\title{
Some results of multidimensional discrete probability measures represented by Euler products
}

\author{
Takahiro Aoyama ${ }^{1}$ and Nobutaka Shimizu ${ }^{2}$ \\ ${ }^{1}$ Faculty of Culture and Education, Saga University, 1 Honjo-machi, Saga 840-8502, Japan \\ ${ }^{2}$ Graduate School of Science and Engineering, Ritsumeikan University, 1-1-1 Noji-higashi, \\ Kusatsu, Shiga 525-8577, Japan \\ E-mail1aoyama@cc.saga-u.ac.jp ${ }^{2}$ ra005065@ed.ritsumei.ac.jp \\ Received February 21, 2014, Accepted March 28, 2014
}

\begin{abstract}
There exist not many treatable multivariable functions with respect to multidimensional discrete distributions. In this paper, we pick up multidimensional finite Euler products and show when they can generate characteristic functions. Moreover, the infinite divisibility of them are studied as well as non-infinite divisibility which are rarely seen in multidimensional discrete case with infinitely many mass points. The relation between series representations of zeta functions is also studied by adjusting to the Shintani zeta type.
\end{abstract}

Keywords characteristic function, Euler product, Lévy measure

Research Activity Group Mathematical Finance

\section{Introduction}

\subsection{Infinite divisibilities}

In mathematical statistics, there exists an important classs of probability distributions. It is defined as follows.

\section{Definition 1 (Infinitely divisible distributions)}

A probability measure on $\mathbb{R}^{d} \mu$ is infinitely divisible if, for any $n \in \mathbb{N}$, there exists a probability measure on $\mathbb{R}^{d}$ $\mu_{n}$ such that

$$
\mu=\mu_{n}^{n *} .
$$

where $\mu_{n}^{n *}$ is the $n$-fold convolution of $\mu_{n}$.

Remark 2 Normal and Poisson distributions are infinitely divisible. This class of distributions is also known to be the marginal distributions of stochastic processes with independent and stationary increments such as Brownian motion and Poisson processes. In mathematical finance, such stochastic processes often appear and we usually call them Lévy processes. We can find the detail of Lévy processes in [1].

Let $\widehat{\mu}(\vec{t}):=\int_{\mathbb{R}^{d}} e^{\mathrm{i}\langle\vec{t}, x\rangle} \mu(d x), \vec{t} \in \mathbb{R}^{d}$, be the characteristic function of a distribution $\mu$, where $\langle\cdot, \cdot\rangle$ is the standard inner product in $\mathbb{R}^{d}$. We also write $a \wedge b=$ $\min \{a, b\}$. Then, we have the following.

Proposition 3 (Lévy-Khintchine representation ( See e.g. [1]) (i) If a probability measure on $\mathbb{R}^{d} \mu$ is infinitely divisible, then

$\widehat{\mu}(\vec{t})=e^{-\frac{1}{2}\langle\vec{t}, A \vec{t}\rangle+\mathrm{i}\langle\gamma, \vec{t}\rangle+\int_{\mathbb{R}^{d}}\left(e^{\mathrm{i}\langle\vec{t}, x\rangle}-1-\frac{\mathrm{i}\langle\vec{t}, x\rangle}{1+|x|^{2}}\right) \nu(d x)}, \quad \vec{t} \in \mathbb{R}^{d}$,

where $A$ is a symmetric nonnegative-definite $d \times d$ matrix, $\gamma \in \mathbb{R}^{d}$, $\nu$ is a measure on $\mathbb{R}^{d}$ satisfying

$$
\nu(\{0\})=0 \quad \text { and } \quad \int_{\mathbb{R}^{d}}\left(|x|^{2} \wedge 1\right) \nu(d x)<\infty .
$$

(ii) The representation given by (1) is unique. (iii) Conversely, if $A$ is a symmetric nonnegative-definite $d \times d$ matrix, $\gamma \in \mathbb{R}^{d}, \nu$ is a measure on $\mathbb{R}^{d}$ satisfying $(2)$, then there exists an infinitely divisible distribution $\mu$ whose characteristic function is given by (1).

Remark 4 The measure $\nu$ is called Lévy measure of an infinitely divisible distribution $\mu$. It represents the constructions of jump type Lévy processes and is often treated in the studies of stochastic models in mathematical finance.

As a generalization of infinitely divisible distributions, we have the following class.

Definition 5 (Quasi-infinitely divisible probability measures (See e.g. [2]) A probability measure on $\mathbb{R}^{d} \mu$ is called quasi-infinitely divisible if, $\widehat{\mu}$ has a form of (1), and corresponding measure $\nu$ is a signed measure on $\mathbb{R}^{d}$ with total variation $|\nu|$ satisfying $\nu(\{0\})=0$ and $\int_{\mathbb{R}^{d}}\left(|x|^{2} \wedge 1\right)|\nu|(d x)<\infty$.

Remark 6 When the measure $\nu$ is not unsigned, the corresponding measure $\mu$ is not infinitely divisible. This fact is applied to show certain distributions not to be infinitely divisible. This class also appears in the studies of convolution powers of distributions. The properties of this class, however, is still not well known. Some results of the relations between multidimensional quasi-infinitle divisibility and multivariable Euler product are given in $[2]$.

\subsection{Zeta functions and distributions}

Zeta functions are one of the main subjects in number theory. The Riemann zeta function is now regarded as the prototype and have a representation by infinite products called the Euler product. They are given as follows. 
Definition 7 (Riemann zeta function, Euler product) For $s=\sigma+\mathrm{i} t, \sigma>1, t \in \mathbb{R}$,

$$
\begin{aligned}
\zeta(s) & =\sum_{n=1}^{\infty} \frac{1}{n^{s}} \\
& =\prod_{p \in \mathbb{P}}\left(1-p^{-s}\right)^{-1},
\end{aligned}
$$

where $\mathbb{P}$ is the collection of all prime numbers. The series (3) is called the Riemann zeta function and the products (4) the Euler product.

Remark 8 The Riemann zeta function $\zeta$ converges absolutely and has no zeros in the region $\sigma>1$. They are now generalized in many ways and often appear in some other fields of mathematics. We can find the basic properties of zeta functions in [3].

There exists an infinitely divisible discrete probability distribution on $\mathbb{R}$ generated by the Riemann zeta function.

Definition 9 (Riemann zeta distribution) For $\sigma$ $>1$, a probability distribution on $\mathbb{R}$ is called the Riemann zeta distribution with a parameter $\sigma$ if it is given by

$$
\operatorname{Pr}\left(X_{\sigma}=-\log n\right)=\frac{n^{-\sigma}}{\zeta(\sigma)}, \quad n \in \mathbb{N} .
$$

Then, we have the following.

Proposition 10 (See, e.g. [4]) The characteristic function $f_{\sigma}$ of the Riemann zeta distribution is given by

$$
f_{\sigma}(t)=\mathbb{E}\left[e^{\mathrm{i} t X_{\sigma}}\right]=\frac{\zeta(\sigma+\mathrm{i} t)}{\zeta(\sigma)}, \quad t \in \mathbb{R} .
$$

Moreover, it is compound Poisson (hence infinitely divisible) and its Lévy measure $N_{\sigma}$ is finite and given by

$$
N_{\sigma}(d x)=\sum_{p \in \mathbb{P}} \sum_{r=1}^{\infty} \frac{p^{-r \sigma}}{r} \delta_{\log p}(d x) .
$$

Remark 11 This proposition is first appeared in [5]. Before that, unnormalized case is introduced as an example in the studies of infinitely many times convolutions of measures in [6].

The Lévy mueasure of the Riemann zeta distribution comes from the Euler product. It means that Euler products may provide us certain new keys and ways of how to treat the jump type Lévy processes. As to obtain multivariable products, the multidimensional polynomial Euler product is introduced in [7] to extend the Riemann zeta distribution to multidimensional cases.

Definition 12 (Multidimensional polynomial Euler produsts [7]) Let $d, k \in \mathbb{N}$ and $\vec{s} \in \mathbb{C}^{d}$. For prime $p,-1 \leq \alpha_{h}(p) \leq 1, \vec{a}_{h} \in \mathbb{R}^{d}$ and $1 \leq h \leq k$, we define multidimensional polynomial Euler products given by

$$
Z_{E}(\vec{s})=\prod_{p \in \mathbb{P}} \prod_{h=1}^{k}\left(1-\alpha_{h}(p) p^{-\left\langle\vec{a}_{h}, \vec{s}\right\rangle}\right)^{-1} .
$$

Our aim is to obtain several definable multidimensional discrete distributions by such products and see whether they are infinitely divisible or not.

\section{Main results}

Denote by

$I D$ : the class of $\mathbb{R}^{2}$-valued infinitely divisible characteristic functions.

$I D^{0}$ : the class of $\mathbb{R}^{2}$-valued quasi-infinitely divisible characteristic functions but non-infinitely divisible characteristic functions.

$N D$ : the class of $\mathbb{R}^{2}$-valued functions not even to be characteristic functions.

Definition 13 Put $s_{1}:=\sigma_{1}+\mathrm{i} t_{1}, s_{2}:=\sigma_{2}+\mathrm{i} t_{2}, \vec{\sigma}:=$ $\left(\sigma_{1}, \sigma_{2}\right)$ and $\vec{t}:=\left(t_{1}, t_{2}\right)$, where $\sigma_{1}, \sigma_{2}>0$ and $t_{1}, t_{2} \in \mathbb{R}$. Then, for $\vec{s}=\left(s_{1}, s_{2}\right)$, we have $\vec{s}=\vec{\sigma}+\mathrm{i} \vec{t}$. For $p \in \mathbb{P}$, define two functions

$$
\begin{aligned}
& g_{p}^{\#}(\vec{\sigma}, \vec{t}):=\frac{1}{\left(1-p^{-\left(\sigma_{1}+\mathrm{i} t_{1}\right)}\right)\left(1-p^{-\left(\sigma_{2}+\mathrm{i} t_{2}\right)}\right)}, \\
& g_{p}^{*}(\vec{\sigma}, \vec{t}):=\frac{1}{\left(1+p^{-\left(\sigma_{1}+\mathrm{i} t_{1}\right)-\left(\sigma_{2}+\mathrm{i} t_{2}\right)}\right)}
\end{aligned}
$$

and corresponding normalized functions

$$
G_{p}^{\#}(\vec{\sigma}, \vec{t}):=\frac{g_{p}^{\#}(\vec{\sigma}, \vec{t})}{g_{p}^{\#}(\vec{\sigma}, \overrightarrow{0})}, \quad G_{p}^{*}(\vec{\sigma}, \vec{t}):=\frac{g_{p}^{*}(\vec{\sigma}, \vec{t})}{g_{p}^{*}(\vec{\sigma}, \overrightarrow{0})} .
$$

By following the definition of the Riemann zeta distribution, it seems to be natural to treat $G_{p}^{\#}$ and $G_{p}^{*}$ to find out whether they can generate characteristic functions of distributions on $\mathbb{R}^{2}$ or not. There are some results of them as follows.

Proposition 14 ([2]) We have the following.
(1) $G_{p}^{\#} \in I D$.
(2) $G_{p}^{*} \in N D$.
(3) $G_{p}^{\#} G_{p}^{*} \in I D^{0}$.

In this paper, we consider the following case.

Definition 15 Let $p_{1}, p_{2} \in \mathbb{P}, \sigma_{1}, \sigma_{2}>0, t_{1}, t_{2} \in \mathbb{R}$. For $s_{1}=\sigma_{1}+\mathrm{i} t_{1}, s_{2}=\sigma_{2}+\mathrm{i} t_{2}, \vec{\sigma}=\left(\sigma_{1}, \sigma_{2}\right), \vec{t}=\left(t_{1}, t_{2}\right)$, we define multidimensional polynomial Euler products $g_{p_{1} p_{2}}^{\sharp}, g_{p_{1} p_{2}}^{*}$ by

$$
\begin{aligned}
g_{p_{1} p_{2}}^{\sharp}(\vec{\sigma}, \vec{t}):= & \frac{1}{\left(1-p_{1}^{-s_{1}}\right)\left(1-p_{1}^{-s_{2}}\right)} \\
& \times \frac{1}{\left(1-p_{2}^{-s_{1}}\right)\left(1-p_{2}^{-s_{2}}\right)}, \\
g_{p_{1} p_{2}}^{*}(\vec{\sigma}, \vec{t}):= & \frac{1}{\left(1+p_{1}^{-s_{1}-s_{2}}\right)\left(1+p_{2}^{-s_{1}-s_{2}}\right)}
\end{aligned}
$$

and their normalized functions

$$
G_{p_{1} p_{2}}^{\sharp}(\vec{\sigma}, \vec{t}):=\frac{g_{p_{1} p_{2}}^{\sharp}(\vec{\sigma}, \vec{t})}{g_{p_{1} p_{2}}^{\sharp}(\vec{\sigma}, 0)}, \quad G_{p_{1} p_{2}}^{*}(\vec{\sigma}, \vec{t}):=\frac{g_{p_{1} p_{2}}^{*}(\vec{\sigma}, \vec{t})}{g_{p_{1} p_{2}}^{*}(\vec{\sigma}, 0)} .
$$

Then, we can also see their behaviors as in the same way as above.

Theorem 16 We have the following.
(1) $G_{p_{1} p_{2}}^{\sharp} \in I D$.
(2) $G_{p_{1} p_{2}}^{*} \in N D$.
(3) $G_{p_{1} p_{2}}^{\sharp} G_{p_{1} p_{2}}^{*} \in I D^{0}$.

Now we add some products. 
Definition 17 Under the same condition as in Definition 15, we define

$$
\begin{aligned}
f_{p_{1} p_{2}}(\vec{\sigma}, \vec{t}) & :=\frac{1}{\left(1-p_{1}^{-s_{1}-s_{2}}\right)\left(1-p_{2}^{-s_{1}-s_{2}}\right)}, \\
g_{p_{1} p_{2}}(\vec{\sigma}, \vec{t}) & :=\prod_{j=1}^{2} \frac{1}{\left(1-p_{j}^{-s_{1}}\right)\left(1-p_{j}^{-s_{2}}\right)\left(1+p_{j}^{-s_{1}-s_{2}}\right)}, \\
h_{p_{1} p_{2}}(\vec{\sigma}, \vec{t}) & :=\prod_{j=1}^{2} \frac{1}{\left(1+p_{j}^{-s_{1}}\right)\left(1+p_{j}^{-s_{2}}\right)\left(1-p_{j}^{-s_{1}-s_{2}}\right)}
\end{aligned}
$$

and their corresponding normalized functions

$$
\begin{aligned}
& F_{p_{1} p_{2}}(\vec{\sigma}, \vec{t}):=\frac{f_{p_{1} p_{2}}(\vec{\sigma}, \vec{t})}{f_{p_{1} p_{2}}(\vec{\sigma}, 0)}, \\
& G_{p_{1} p_{2}}(\vec{\sigma}, \vec{t}):=\frac{g_{p_{1} p_{2}}(\vec{\sigma}, \vec{t})}{g_{p_{1} p_{2}}(\vec{\sigma}, 0)}, \\
& H_{p_{1} p_{2}}(\vec{\sigma}, \vec{t}):=\frac{h_{p_{1} p_{2}}(\vec{\sigma}, \vec{t})}{h_{p_{1} p_{2}}(\vec{\sigma}, 0)} .
\end{aligned}
$$

Then, we obtain the following theorems.

\section{Theorem 18}

(1) $F_{p_{1} p_{2}} \in I D$.

(2) $G_{p_{1} p_{2}} \in I D^{0}$.

(3) $H_{p_{1} p_{2}} \in N D$.

\section{Theorem 19}

(1) $F_{p_{1} p_{2}} G_{p_{1} p_{2}} \in I D$.

(2) $G_{p_{1} p_{2}} H_{p_{1} p_{2}} \in I D$.

(3) $H_{p_{1} p_{2}} F_{p_{1} p_{2}} \in N D$.

Remark 20 As further results, behaviors of products of these functions are studied in Aoyama and Shimizu [9].

We need the following facts to show our results.

Proposition 21 Let $\mu$ be a probability measure on $\mathbb{R}^{d}$. Then, $|\widehat{\mu}(\vec{t})| \leq 1$ for any $\vec{t} \in \mathbb{R}^{d}$, where $\widehat{\mu}$ is the characteristic function of $\mu$.

For $n \in \mathbb{N}$, let $\vec{a}_{k}=\psi_{k} \vec{a},(1 \leq k \leq n)$ be vectors on $\mathbb{R}^{d}$ where $\psi_{1}, \ldots, \psi_{n}$ are algebraic real numbers. Then we call $\vec{a}_{1}, \ldots, \vec{a}_{n}$ are linearly dependent but linearly independent over $\mathbb{Q}$, if $c_{1} \psi_{1}+\cdots+c_{n} \psi_{n}=0$ is equivalently $c_{1}=\cdots=c_{n}=0,\left(c_{1}, \ldots, c_{n} \in \mathbb{Q}\right)$. Denote by

LI: Linearly independent,

LR: Linearly dependent but linearly independent over $\mathbb{Q}$.

Put $f_{\vec{\sigma}}(\vec{t}):=Z_{E}(\vec{\sigma}+\mathrm{i} \vec{t}) / Z_{E}(\vec{\sigma})$. The following lemma is one of the keys for our proof coming later.

Lemma 22 ([7]) Suppose that $\vec{a}_{1}, \ldots, \vec{a}_{k}$ satisfy LI or LR. If there exists a set of pairs of $h$ and $q,(1 \leq h \leq$ $k, q \in \mathbb{P})$ such that $\alpha_{h}(q)<0$, then there exists $\overrightarrow{t_{0}} \in \mathbb{R}^{d}$ such that $\left|f_{\vec{\sigma}}\left(\vec{t}_{0}\right)\right|>1$.

They also gave the following.

Proposition 23 ([7]) Suppose that $\vec{a}_{1}, \ldots, \vec{a}_{k}$ satisfy LI or LR. Then $f_{\vec{\sigma}}$ is a characteristic function if and only if $\alpha_{h}(p) \geq 0$ for all $1 \leq h \leq k$ and $p \in \mathbb{P}$. Moreover, $f_{\vec{\sigma}}$ is a compound Poisson characteristic function with a finite Lévy measure $N_{\vec{\sigma}}$ on $\mathbb{R}^{d}$ given by

$$
N_{\vec{\sigma}}(d x)=\sum_{p \in \mathbb{P}} \sum_{r=0}^{\infty} \sum_{h=1}^{k} \frac{1}{r} \alpha_{h}(p)^{r} p^{-r\left\langle\vec{a}_{h}, \vec{\sigma}\right\rangle} \delta_{r \log p \vec{a}_{h}}(d x) .
$$

Now we give the proof of Theorem 16 .

Proof of Theorem 16 (1) It can be proved by following Proposition 23.

(QED)

Proof of Theorem 16 (2) We have that there exists $\vec{t}_{0}$ such that $\left|G_{p_{1} p_{2}}^{*}\left(\vec{t}_{0}\right)\right|>1$ by Lemma 22 . This contradicts the fact as in Proposition 21.

(QED)

We need the followings before we prove Theorem 16 (3).

Definition 24 (Multidimensional Shintani zeta function [8]) Let $d, m, r \in \mathbb{N}, \vec{s} \in \mathbb{C}^{d}$ and $\left(n_{1}, \ldots, n_{r}\right) \in \mathbb{Z}_{>0}^{r}$. For $\lambda_{l j}, u_{j}>0, \vec{c}_{l} \in \mathbb{R}^{d}$, where $1 \leq$ $j \leq r$ and $1 \leq l \leq m$, and a function $\theta\left(n_{1}, \ldots, n_{r}\right) \in \mathbb{C}$ satisfying $\left|\theta\left(n_{1}, \ldots, n_{r}\right)\right|=O\left(\left(n_{1}+\cdots+n_{r}\right)^{\varepsilon}\right)$, for any $\varepsilon>0$, we define a multidimensional Shintani zeta function $Z_{S}(\vec{s})$ given by

$$
\sum_{n_{1}, \ldots, n_{r}=0}^{\infty} \frac{\theta\left(n_{1}, \ldots, n_{r}\right)}{\prod_{l=1}^{m}\left[\sum_{j=1}^{r} \lambda_{l j}\left(n_{j}+u_{j}\right)\right]^{\left\langle\vec{c}_{l}, \vec{s}\right\rangle}} .
$$

The series $Z_{S}(\vec{s})$ converges absolutely in the region $\min _{1 \leq l \leq m}\left\langle\vec{c}_{l}, \vec{\sigma}\right\rangle>r / m$ (see, Aoyama and Nakamura $[8])$, which we denote by $\mathrm{D}_{\mathrm{S}}$. Suppose that $\theta\left(n_{1}, \ldots, n_{r}\right)$ is non-negative or non-positive definite, then we have the following class of distribution on $\mathbb{R}^{d}$.

Definition 25 (Multidimensional Shintani zeta distribution [8]) For each $\vec{\sigma} \in \mathrm{D}_{\mathrm{S}}$, a probability measure $\mu_{\vec{\sigma}}$ on $\mathbb{R}^{d}$ is called a multidimensional Shintani zeta distribution, if for all $\left(n_{1}, \ldots, n_{r}\right) \in \mathbb{Z}_{\geq 0}^{r}$,

$$
\begin{array}{r}
\mu_{\vec{\sigma}}\left(\left\{-\sum_{l=1}^{m} c_{l 1} \log \left(\sum_{k=1}^{r} \lambda_{l k}\left(n_{k}+u_{k}\right)\right)\right.\right. \\
\left.\left.\ldots,-\sum_{l=1}^{m} c_{l d} \log \left(\sum_{k=1}^{r} \lambda_{l k}\left(n_{k}+u_{k}\right)\right)\right\}\right) \\
=\frac{\theta\left(n_{1}, \ldots, n_{r}\right)}{Z_{S}(\vec{\sigma})} \prod_{l=1}^{m}\left[\sum_{k=1}^{r} \lambda_{l k}\left(n_{k}+u_{k}\right)\right]^{-\left\langle\vec{c}_{l}, \vec{\sigma}\right\rangle} .
\end{array}
$$

Then its characteristic function $f_{\vec{\sigma}}$ is given by

$$
f_{\vec{\sigma}}(\vec{t}):=\int_{\mathbb{R}^{d}} e^{\mathrm{i}\langle\vec{t}, x\rangle} \mu_{\vec{\sigma}}(d x)=\frac{Z_{S}(\vec{\sigma}+\mathrm{i} \vec{t})}{Z_{S}(\vec{\sigma})}, \quad \vec{t} \in \mathbb{R}^{d} .
$$

Now we prove Theorem $16(3)$.

Proof of Theorem 16 (3) First we show that $G_{p_{1} p_{2}}^{\sharp} G_{p_{1} p_{2}}^{*}$ is a characteristic function. We have

$$
\begin{aligned}
& g_{p_{1} p_{2}}^{\sharp}(\vec{\sigma}, \vec{t}) g_{p_{1} p_{2}}^{*}(\vec{\sigma}, \vec{t}) \\
& \quad=\prod_{j=1}^{2} \frac{1}{\left(1-p_{j}^{-2 s_{1}-2 s_{2}}\right)} \times \frac{\left(1-p_{j}^{-s_{1}-s_{2}}\right)}{\left(1-p_{j}^{-s_{1}}\right)\left(1-p_{j}^{-s_{2}}\right)} .
\end{aligned}
$$

For any $X, Y$ with $|X|,|Y|<1$, we also have $1 /(1-X)=$ $\sum_{n=0}^{\infty} X^{n}$ and $(1-X Y) /[(1-X)(1-Y)]=1 /(1-X)+$ $1 /(1-Y)-1=1+\sum_{n=1}^{\infty}\left(X^{n}+Y^{n}\right)$. Therefore, we 
obtain

$$
\begin{aligned}
g_{p_{1} p_{2}}^{\sharp}(\vec{\sigma}, \vec{t}) g_{p_{1} p_{2}}^{*}(\vec{\sigma}, \vec{t}) & \\
= & \sum_{l_{1}=0}^{\infty} \frac{1}{p_{1}^{2 l_{1}\left(s_{1}+s_{2}\right)}}\left(1+\sum_{m_{1}=1}^{\infty} \frac{1}{p_{1}^{m_{1} s_{1}}}+\sum_{n_{1}=1}^{\infty} \frac{1}{p_{1}^{n_{1} s_{2}}}\right) \\
& \times \sum_{l_{2}=0}^{\infty} \frac{1}{p_{2}^{2 l_{2}\left(s_{1}+s_{2}\right)}}\left(1+\sum_{m_{2}=1}^{\infty} \frac{1}{p_{2}^{m_{2} s_{1}}}+\sum_{n_{2}=1}^{\infty} \frac{1}{p_{2}^{n_{2} s_{2}}}\right) \\
= & \left(\sum_{l_{1}, m_{1}, n_{1}=1}^{\infty} \frac{A_{1}\left(l_{1}\right) A_{1}\left(m_{1}, n_{1}\right)}{l_{1}^{s_{1}+s_{2}} m_{1}^{s_{1}} n_{1}^{s_{2}}}\right) \\
& \times\left(\sum_{l_{2}, m_{2}, n_{2}=1}^{\infty} \frac{A_{2}\left(l_{2}\right) A_{2}\left(m_{2}, n_{2}\right)}{l_{2}^{s_{1}+s_{2}} m_{2}^{s_{1}} n_{2}^{s_{2}}}\right),
\end{aligned}
$$

where, for $a, b, c \in \mathbb{N}$ and $j=1,2$,

$$
\begin{aligned}
& A_{j}\left(l_{j}\right):= \begin{cases}1 & l_{j}=1, p_{j}^{2 a}, \\
0 & \text { otherwise },\end{cases} \\
& A_{j}\left(m_{j}, n_{j}\right):= \begin{cases}1 & \left(m_{j}, n_{j}\right)=(1,1),\left(1, p_{j}^{b}\right),\left(p_{j}^{c}, 1\right), \\
0 & \text { otherwise. }\end{cases}
\end{aligned}
$$

We can see that both of $\sum_{l_{1}, m_{1}, n_{1}=1}^{\infty} A_{1}\left(l_{1}\right) A_{1}\left(m_{1}\right.$, $\left.n_{1}\right) /\left(l_{1}^{s_{1}+s_{2}} m_{1}^{s_{1}} n_{1}^{s_{2}}\right)$ and $\sum_{l_{2}, m_{2}, n_{2}=1}^{\infty} A_{2}\left(l_{2}\right) A_{2}\left(m_{2}, n_{2}\right) /($ $\left.l_{2}^{s_{1}+s_{2}} m_{2}^{s_{1}} n_{2}^{s_{2}}\right)$ belong to multidimensional Shintani zeta function with above nonnegative sequences as character $\theta$. Thus, $G_{p_{1} p_{2}}^{\sharp} G_{p_{1} p_{2}}^{*}$ is a characteristic function of multidimensional Shintani zeta distribution.

Next, we show that $G_{p_{1} p_{2}}^{\sharp} G_{p_{1} p_{2}}^{*}$ is quasi but not infinitely divisible. We have that

$$
\begin{aligned}
& \log G_{p_{1} p_{2}}^{\sharp}(\vec{\sigma}, \vec{t}) G_{p_{1} p_{2}}^{*}(\vec{\sigma}, \vec{t}) \\
&=\sum_{j=1}^{2}\left(\log \left(1-p_{j}^{-\sigma_{1}}\right)-\log \left(1-p_{j}^{-\sigma_{1}-\mathrm{i} t_{1}}\right)\right. \\
&+\log \left(1-p_{j}^{-\sigma_{2}}\right)-\log \left(1-p_{j}^{-\sigma_{2}-\mathrm{i} t_{2}}\right) \\
&+\log \left(1+p_{j}^{-\sigma_{1}-\sigma_{2}}\right) \\
&\left.\quad-\log \left(1+p_{j}^{-\sigma_{1}-\mathrm{i} t_{1}-\sigma_{2}-\mathrm{i} t_{2}}\right)\right) \\
&=\sum_{j=1}^{2}\left(\sum_{r=1}^{\infty} \frac{1}{r} p_{j}^{-r \sigma_{1}}\left(p_{j}^{-r i t_{1}}-1\right)\right. \\
& \quad+\sum_{r=1}^{\infty} \frac{1}{r} p_{j}^{-r \sigma_{2}}\left(p_{j}^{-r i t_{2}}-1\right) \\
&\left.\quad+\sum_{r=1}^{\infty} \frac{(-1)^{r}}{r} p_{j}^{-r\left(\sigma_{1}+\sigma_{2}\right)}\left(p_{j}^{-r \mathrm{i}\left(t_{1}+t_{2}\right)}-1\right)\right) \\
&=\int_{\mathbb{R}^{2}}\left(e^{\mathrm{i}\langle\vec{t}, x\rangle}-1\right) N_{\vec{\sigma}}^{G_{p_{1} p_{2}}^{\sharp} G_{p_{1} p_{2}}^{*}}(d x),
\end{aligned}
$$

where

$$
\begin{aligned}
& N_{\vec{\sigma}}^{G_{p_{1} p_{2}}^{\sharp} G_{p_{1} p_{2}}^{*}}(d x) \\
& :=\sum_{j=1}^{2}\left(\sum_{r=1}^{\infty} \frac{1}{r} p_{j}^{-r \sigma_{1}} \delta_{r \log p_{j}(1,0)}(d x)\right.
\end{aligned}
$$

$$
\begin{aligned}
& +\sum_{r=1}^{\infty} \frac{1}{r} p_{j}^{-r \sigma_{2}} \delta_{r \log p_{j}(0,1)}(d x) \\
& \left.+\sum_{r=1}^{\infty} \frac{(-1)^{r}}{r} p_{j}^{-r\left(\sigma_{1}+\sigma_{2}\right)} \delta_{r \log p_{j}(1,1)}(d x)\right) .
\end{aligned}
$$

The measure $N_{\vec{\sigma}}^{G_{p_{1} p_{2}}^{\sharp}} G_{p_{1} p_{2}}^{*}$ is a quasi-Lévy measure on $\mathbb{R}^{2}$ with negative components since the 3 rd and 6 th term are negative signed measures which cannot be canceled by the other terms and we easily obtain

$$
\int_{\mathbb{R}^{2}}\left|N_{\vec{\sigma}}^{G_{p_{1} p_{2}}^{\sharp} G_{p_{1} p_{2}}^{*}}\right|(d x)<\infty .
$$

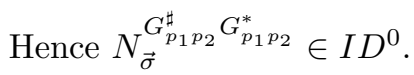

(QED)

The proofs of Theorem 18, 19 and further results are given in [9]. Such properties may provide us new methods to construct stochastic models in mathematical finance.

\section{Acknowledgments}

The authors would like to express sincere appreciations to Professor Jirô Akahori for his variable comments. This work was partially supported by JSPS KAKENHI Grant Numbers 23330190, 24340022, 23654056 and 25285102.

\section{References}

[1] K. Sato, Lévy Processes and Infinitely Divisible Distributions, Cambridge Univ. Press, Cambridge, 1999.

[2] T. Aoyama and T. Nakamura, Behaviors of multivariable finite Euler products in probabilistic view, Math. Nachr., 286 (2013), 1691-1700.

[3] T. M. Apostol, Introduction to Analytic Number Theory, Undergraduate Texts in Mathematics, Springer-Verlag, New York, 1976.

[4] B. V. Gnedenko and A. N. Kolmogorov, Limit Distributions for Sums of Independent Random Variables (Translated from the Russian by Kai Lai Chung), Addison-Wesley, London, 1968.

[5] A. Ya. Khinchine, Limit Theorems for Sums of Independent Random Variables (in Russian), GONTI, Moscow, 1938.

[6] B. Jessen and A. Wintner, Distribution functions and the Riemann zeta function, Trans. Amer. Math. Soc., 38 (1935), 48-88.

[7] T. Aoyama and T. Nakamura, Multidimensional polynomial Euler products and infinitely divisible distributions on $\mathbb{R}^{d}$, submitted, http://arxiv.org/abs/1204.4041.

[8] T. Aoyama and T. Nakamura, Multidimensional Shintani zeta functions and zeta distributions on $\mathbb{R}^{d}$, Tokyo J. Math., 36 (2013), 521-538.

[9] T. Aoyama and N. Shimizu, Properties of multidimensional discrete distributions having Euler products, preprint. 